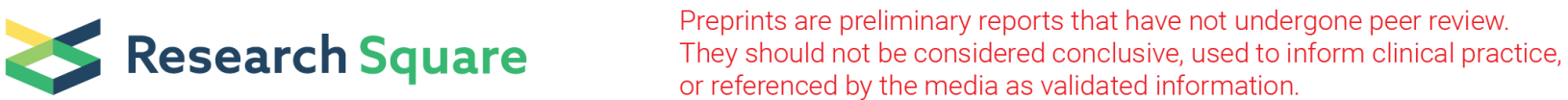 \\ DenseCapsNet: Detection of COVID-19 X-ray Images Using a Capsule Network
}

\section{Hao Quan}

College of Medicine and Biological Information Engineering, Northeastern University, Shenyang 110001, China

\section{Xiaosong Xu}

College of Medicine and Biological Information Engineering, Northeastern University, Shenyang 110001, China

\section{Tingting Zheng}

College of Medicine and Biological Information Engineering, Northeastern University, Shenyang 110001, China

\section{Zhi Li}

Department of Medical Oncology, the First Hospital of China Medical University, Shenyang 110001, China

\section{Mingfang Zhao}

Department of Medical Oncology, the First Hospital of China Medical University, Shenyang 110001, China

\section{Xiaoyu Cui ( $\nabla$ cuixy@bmie.neu.edu.cn )}

College of Medicine and Biological Information Engineering, Northeastern University, Shenyang 110001, China

\section{Method Article}

Keywords: COVID-19, X-rays, Deep Learning, Artificial intelligence

Posted Date: October 2nd, 2020

DOl: https://doi.org/10.21203/rs.3.rs-84220/v1

License: (1) This work is licensed under a Creative Commons Attribution 4.0 International License. Read Full License 


\section{Abstract}

Objective: A deep learning framework for detecting COVID-19 is developed, and a small amount of chest $\mathrm{X}$-ray data is used to accurately screen COVID-19.

Methods: In this paper, we propose a deep learning framework that integrates convolution neural network and capsule network. DenseNet and CapsNet fusion are used to give full play to their respective advantages, reduce the dependence of convolution neural network on a large amount of data, and can quickly and accurately distinguish COVID-19 from Non-COVID-19 through chest X-ray imaging.

Results: A total of 1472 chest X-ray COVID-19 and non-COVID-19 images are used, this method can achieve an accuracy of $99.32 \%$ and a precision of $100 \%$, with $98.55 \%$ sensitivity and $100 \%$ specificity.

Conclusion: These results show that the deep fusion neural network DenseCapsNet has good performance in novel coronavirus pneumonia X-ray detection. We also prove through experiments that the detection performance of DenseCapsNet is not affected fundamentally by a lack of data augmentation and pre-training.

\section{Key Points}

- We fuse DenseNet and CapsNet to form a new deep learning framework DenseCapsNet, which can quickly and accurately detect COVID-19 chest $X$-ray.

- Through a large number of experiments, DenseCapsNet is proved to be meaningful for large-scale screening of COVID-19.

- Reduce the dependence of convolution neural network on big data through capsule network.

\section{Introduction}

In December 2019, researchers discovered a new type of febrile respiratory disease caused by severe acute respiratory syndrome coronavirus type 2 (SARS-CoV-2), which spread rapidly all over the world. The novel coronavirus pneumonia was officially named Corona Virus Disease 2019 (COVID-19) [1] by the World Health Organization (WHO) on February 11, 2020. By the end of August 2020, the number of COVID-19 cases exceeded 25 million cases, and the number of deaths exceeded 800,000 [2], which has seriously hindered economic development and cultural exchange around the world and poses a serious threat to human health.

The reverse transcription-polymerase chain reaction (RT-PCR) can detect pre-symptomatic patients and identify infected patients early, which is the "gold standard" of novel coronavirus tests [3-5]. However, it requires high-performance detection equipment or platforms, and high-sensitivity RT-PCR instruments are expensive. In addition, nucleic acid detection takes a long time. Considering the transportation of samples and the backlog of samples, the results can usually be reported within 24 hours at the earliest. 
Compared with nucleic acid tests, radiological examinations (CT or X-ray) also have some visual markers, such as frosted glass turbidity and bilateral radiation abnormalities [6][7], that can be used to distinguish COVID-19 infection from non-COVID-19 infection. Because of its high sensitivity, CT is widely used in the auxiliary diagnosis of COVID-19 [5][8]. However, novel coronavirus's high infection rate has led to its rapid spread worldwide. Low-cost rapid detection technology is crucial to the screening of novel coronavirus pneumonia, especially in low-developed areas. Although chest X-ray is helpful for the early detection of COVID-19, there are many kinds of viral pneumonia that have certain similarities, which makes it difficult for inexperienced radiologists to accurately distinguish other viral pneumonia from COVID-19. It has been reported that the sensitivity of using X-ray to check for COVID-19 is only approximately 69\%[9]. If the performance of X-ray detection of COVID-19 can be significantly improved, this will be of great significance.

With the successful application of artificial intelligence technology in the medical field, one excellent method is to use medical image processing and deep learning technology to assist in detecting and diagnosing medical images [10]. Deep learning technology can reveal many inconspicuous image features in medical images. Convolutional neural networks (CNNs) for deep feature extraction have been widely used in the research field. Of course, in-depth learning technology for COVID-19 detection has also been actively explored[11-16]. Linda Wang et al. [11] proposed a deep CNN COVID-Net, which can be used for COVID-19 detection, and achieved $93.3 \%$ overall accuracy in the dataset COVIDx that the authors proposed; the sensitivity of COVID-19 detection reached $91 \%$.

At present, the $\mathrm{CNN}$, as an excellent feature extractor, is the method used by most researchers who use deep learning technology to detect COVID-19. However, CNNs have some defects, such as being unable to capture the spatial relationships of features and being unable to recognize pictures with rotations or other transformations. In 2017, Geoffrey E. Hinton et al. [17] introduced the capsule network in detail. The capsules in the capsule network are composed of a group of related neurons, which represent various attributes of a specific entity, such as posture, texture features, and tone. Capsule networks can fully capture image features, postures and spatial relationships by means of neuron "packaging", so capsules can detect certain types of patterns and reduce the dependence of the network on large datasets. The capsule network has been proven to be a good substitute for CNNs. This is truly an exciting achievement. Parnian Afshar et al. [18] aimed to use a capsule network to detect COVID-19 and proposed COVID-CAPS, and they achieved excellent results, with an overall accuracy of $95.7 \%$ and sensitivity of $90 \%$. The results proved that capsule network detection of COVID-19 was feasible.

Using deep learning technology to analyse medical image data itself tests the quality and quantity of datasets, and it is difficult to obtain a large amount of image data with good quality when a new epidemic suddenly occurs. In this work, we focus on solving the problem of training excellent detection models on small COVID-19 datasets. Our network structure takes the capsule network as the main body, uses DenseNet[19] as the feature extractor, and proposes a deep learning model DenseCapsNet for COVID-19 detection. We have proved through experiments that DenseCapsNet can effectively train an excellent COVID-19 detection model in a limited dataset. Compared with COVID-19 detection models 
using the same data, our proposed network has better overall accuracy and sensitivity and can detect COVID-19 more accurately.

\section{Materials And Methods}

\section{A. Dataset Description}

In this work, we used COVIDx(As shown in Fig.1), the COVID-19 dataset collated by Linda Wang et al. [11]The dataset consists of 13,975 chest X-rays of 13,870 patients. The disease types of COVIDx dataset are mainly divided into four categories: normal (without pneumonia infection), bacterial pneumonia, viral pneumonia, and COVID-19.

To compare COVID-CAPS[18] and COVID-Net[11] methods, we extracted some data from the COVIDx dataset and divided them into two parts: dataset A, containing 1475 chest X-rays, and dataset B, containing 2260 chest $X$-rays. Dataset $A$ is designed to train the binary classification model. Referring to the central idea of COVID-CAPS, we divide dataset A into two categories, positive (COVID-19) and negative (other viral pneumonia, bacterial pneumonia and normal); 687 are positive $\mathrm{X}$-rays and 785 are negative X-rays. Dataset B is designed for comparison with the COVID-Net method. Referring to the central idea of COVID-Net, the dataset is divided into three categories, COVID-19, pneumonia and normal; it includes 687 chest X-rays in the COVID-19 category, 785 in the pneumonia category and 785 in the normal category. We divide the above two datasets into training sets and test sets according to a ratio of 7: 3. Dataset A has 1030 chest $X$-rays for model training and 445 chest $X$-rays for model testing. A total of 1580 chest $X$-rays were used for model training, and 680 chest $X$-rays were used for model testing in dataset $\mathrm{B}$.

\section{B. Data Augmentation}

The method of data augmentation should be as random as possible to generate more meaningful training data. In our study, we randomly rotated, vertically flipped and cut the training data to create new examples. We also regularize the data to speed up the model fitting process and ultimately improve the generalization of the model.

\section{DenseCapsNet}

Feature Extraction Using Convolutional Neural Networks DenseNet can make information flow fully, reuse features, strengthen feature propagation and reduce the number of parameters through its densely connected features. These characteristics enable DenseNet to achieve better results than ResNet[20] under the same parameter quantity in less time. We use DenseNet121 after ImageNet[21] pre-training to prevent the effect of random model initialization on the model fitting ability and to ensure that the model can achieve better results on a smaller dataset to the greatest extent possible. DenseNet121 is the simplest type of dense convolution network and has the smallest number of parameters. The network structure is shown in Fig.3. We reduced the chest X-ray data to a unified size, enhanced it with data and 
then converted it into tensors. After normalization, we input it into the DenseNet121 network architecture. Using DenseNet121's excellent feature extraction and feature reuse capabilities, we retained all the kinds of detailed features of the data as much as possible and finally output 1024 features.

Capsule Network The architecture of the capsule network is relatively clear and mainly consists of a series of capsule layers [17]. The capsule layer of the capsule network is composed of multiple capsules, and each capsule is composed of a group of neurons. The primary capsule layer is preceded by a 512channel convolution layer, which is used to filter the 512 features output by DenseNet 121 . After the reshaping operation, a primary capsule layer is formed. Dynamic routing is adopted between the primary capsule layer and the convolution capsule layer to route the output vector to all possible parent nodes.

Loss function For the loss function of the capsule network, we use the spread loss function, which can reduce the sensitivity of training to the model initialization and super-parameters, and $a_{t}$ is the target class activation. If the distance between the activation error classes $a_{i}$ and $a_{t}$ is less than Margin $(m)$, the loss function will punish the model according to $\left(m-\left(a_{t}-a_{i}\right)\right)^{2}$.

$$
L_{i}=\max \left(0, m-\left(a_{t}-a_{i}\right)\right)^{2}, L=\sum_{i \neq t} L_{i}
$$

The initial value of Margin is set to 0.2. During the training process, the value of Margin increases by 0.1 to 0.9 per iteration, which can prevent early capsule death.

DenseCapsNet Network Structure The structure of DenseCapsNet that we propose is shown in Fig.2. Above, we briefly introduced DenseNet121 and explained the leading position of DenseNet121 in a CNN. Using DenseNet121 as a feature extractor is a good choice. DenseCapsNet is mainly composed of DenseNet121 and CapsNet so that it gives full play to the advantages of CNNs and capsule networks. First, we retain the feature extraction part of the CNN DenseNet121 (excluding the linear layer) to extract the features from novel coronavirus pneumonia chest X-ray data. We input novel coronavirus pneumonia 3D X-ray data and adjust the data to a unified size. DenseNet 121 outputs 1024 feature maps and transmits them to the first convolution layer of the capsule network, which filters the above features to 512 and reshapes them into vectors to be transmitted to the main capsule layer. Finally, the capsule layer outputs the instantiated parameters containing normal and COVID-19 images, and the vector lengths represent the probability of the occurrence of each category.

\section{Results}

\section{A. Model Parameter Setting and Evaluation Method}

We set the iteration number as 30 , the learning rate as 0.0001 , the optimizer as ADAM, and the dynamic routing iteration number in the capsule network as 3 . All experiments were carried out on Core i3 8th Gen, with 16GB RAM and TITAN Xp. All our implementations are performed in PyTorch. 
We adopt Accuracy , Precision, Sensitivity and Specificity as the model evaluation metrics, which are used by most researchers on novel coronavirus pneumonia $[16,22,23]$ and have authority. We also give the confusion matrix of the model, which can fully demonstrate the prediction ability of our model.

\section{B. Experimental Design and Results}

In this work, we designed three groups of experiments to show the performance advantages of DenseCapsNet in detecting COVID-19. The experimental design scheme is shown in Fig.4. All our experiments were repeated three times and averaged to avoid accidental occurrences.

Experiment I: The ResNet50 and DenseNet121 neural networks are classical networks among CNNs. We chose the above two networks as representatives of CNNs. Then, we combined the ResNet50 and DenseNet121 networks as feature extraction layers with capsule networks to generate ResCapsNet and DenseCapsNet, respectively. We used dataset A to train the above four networks, and all used data augmentation and pre-training. The experimental results are shown in Table 1.

Experiment II: We wanted to explore whether data augmentation and pre-training would have a significant impact on our proposed DenseCapsNet. We still used dataset A as the training data and conducted three experiments: the first time we only used data augmentation; only pre-training was used the second time. The third time, neither data augmentation nor pre-training was used. To fully prove DenseCapsNet's binary classification performance advantages, we also compared the performance indexes of COVIDCAPS, and the experimental results are shown in Table 2.

Experiment III: We trained DenseCapsNet with dataset B to construct a three-group classification network that could accurately detect COVID-19, non-COVID-19 pneumonia and a normal chest X-ray. The aim was to verify the recognition ability of DenseCapsNet for COVID-19 chest X-rays and compare it with that of COVID-Net. The sensitivity and positive predictive value (PPV) of DenseCapsNet for each infection type are shown in Table 3.

\section{Discussion}

Through the above experimental results, DenseCapsNet deep learning framework has high sensitivity to COVID-19, and the network framework can indeed obtain sufficient features on small datasets to realize accurate detection of COVID-19. The results obtained in experiment I can show that capsule network can significantly improve the performance of CNN in COVID-19 chest X-ray detection. Experiment II proves that DenseCapsNet does not rely on data augmentation and pre-training, and the framework is robust. We also want to test DenseCapsNet's ability to detect three classifications (Normal, Non-COVID-19 and COVID-19), through Experiment III and compared with COVID-Net, the framework does achieve optimal performance. Next, we will fully discuss the above three groups of comparative experiments.

Experiment I: According to Table 1, when using a traditional CNN alone to detect COVID-19 chest X-rays, the detection abilities of DenseNet121 and ResNet50 can be compared. Other than precision, the indexes 
are significantly different, and the sensitivity reaches a difference of nearly $20 \%$. This is very important; the higher the sensitivity is, the lower the possibility of the false detection of COVID-19 patients. According to the confusion matrix of Experiment I in Fig. 5, we can clearly see that the two CNN frameworks can achieve good detection for normal $X$-ray images and have similar performance, but in the detection of COVID-19, the detection capability of the two network frameworks is not satisfactory. DenseNet121 detected 43 COVID-19 patients as normal, ResNet50 detected 84 COVID-19 patients as normal, and the number of false detections by ResNet50 was almost twice that of DenseNet121. This fully proves that DenseNet121 is better than ResNet50 in detecting COVID-19. This may be due to the deepening of the network level; DenseNet 121 can mine deeper features. DenseNet 121 also has excellent feature transfer and feature reuse functions, which make it superior to ResNet 50. However, the performance of DenseNet121 is not satisfactory. In Table 1, we can also clearly see that the combination of a CNN and capsule network is significantly better than the CNN alone in detecting COVID-19. We combine DenseNet121 with CapsNet to form DenseCapsNet, combine ResNet50 with CapsNet to form ResCapsNet, and test the ability of these two models to detect COVID-19. The results of these two frameworks are better than those of the ResNet50 and DenseNet121 models used alone. As can be seen from that confusion matrix of Experiment I in Fig. 5, both DenseCapsNet and ResCapsNet have high sensitivity in detecting COVID-19. ResCapsNet misdetects 5 COVID-19 patients, while DenseCapsNet misdetects only 3 patients, so DenseCapsNet is better. The purpose of our work is not only to improve the detection sensitivity of COVID-19 but also to measure the training time. Under the same parameters and the same test equipment, we use the same dataset to train ResCapsNet and DenseCapsNet. The number of epochs is 30; ResCapsNet takes 12 hours, 47 minutes and 56 seconds, and DenseCapsNet takes 6 hours, 3 minutes and 24 seconds. The training time of DenseCapsNet is less than half that of ResCapsNet, and DenseCapsNet also has significant advantages in terms of the training time.

Experiment II: When we decided to use the DenseNet121 network as the CNN part of the composite network, we performed another group of experiments on the obtained DenseCapsNet. We wanted to explore whether data augmentation and pre-training would have some influence on DenseCapsNet and to compare it with COVID-CAPS. The experimental results are shown in Table 2, and the confusion matrix is shown in Experiment II in Fig. 5. According to the above chart, we can clearly see that the impact of data augmentation and pre-training on DenseCapsNet's detection capability is very small; that is,

DenseCapsNet can achieve excellent results without relying on data augmentation and pre-training. When we use the same dataset to train DenseCapsNet without data augmentation and pre-training, the number of iterations is 30 , and the time taken is 3 hours, 40 minutes and 35 seconds. The substantial shortening of the training time is mainly due to the elimination of data augmentation. We can increase the batch size to speed up training. We used the same type of dataset as COVID-CAPS, and the amount of data we used was smaller than that for COVID-CAPS. As seen from Table 2, DenseCapsNet's overall performance is better than that of COVID-CAPS, and it can detect COVID-19 patients more accurately, which is of great significance for reducing the screening pressure of medical workers and can significantly increase the efficiency of early diagnosis. 
Experiment III: Through the first two experiments, we show that DenseCapsNet can indeed screen COVID19 patients from chest X-rays of unknown patients. If DenseCapsNet can detect normal images in more detail, the detection of other pneumonia and COVID-19 will be even better. In this way, COVID-19 and other pneumonia could be directly screened from massive chest X-ray data. Doctors could then isolate COVID19 patients and treat them in a targeted way. Other pneumonia patients would not need separate isolation but centralized treatment. Healthy people would go home for isolation to reduce hospital pressure. We used the same type of dataset B as COVID-Net but with a small amount of data. In Table 3, DenseCapsNet is shown to be significantly more sensitive to the three types of chest X-ray data than COVID-Net, especially for COVID-19, reaching an excellent result of $99.52 \%$. As can be seen from that confusion matrix of Experiment III of Fig. 5 DenseCapsNet correctly detected almost all chest X-rays of COVID-19 patients (only one case was misdetected). However, for the PPV in Table 3, DenseCapsNet's prediction for COVID-19 patients is slightly lower than that of COVID-Net, which means DenseCapsNet predicted some non-COVID-19 chest X-rays as COVID-19. A total of 6 cases were misdiagnosed as COVID19 , of which 5 cases were patients with other pneumonia, mainly because other pneumonia and COVID19 have a high similarity on chest X-ray images. The detection capability of DenseCapsNet may be further improved by increasing the data volume of the training datasets.

In this work, the combination of a $\mathrm{CNN}$ and capsule network is shown to be better than using a CNN alone in the detection of COVID-19. However, the combination of networks also brings some problems, such as the increase of the number of network parameters and the extension of the training time. At present, the number of COVID-19 patients is continuously increasing, and we need a more robust and faster network to perform mass screening of COVID-19. Our work can relieve the pressure of the mass screening of COVID-19 patients to a certain extent, but we will also strive to build better models to cope with the daily spread of the epidemic.

\section{Conclusion}

In this paper, we propose a deep learning framework for COVID-19 detection based on DenseNet121 and CapsNet. We have taken full advantage of CNNs for feature extraction and of capsule networks for packaging features into capsules so that they do not rely on large datasets. Using only 480 COVID-19 chest $\mathrm{X}$-rays and 550 normal chest $\mathrm{X}$-rays, we achieved excellent results, with the accuracy reaching $99.32 \%$. We have proven that the combination of a CNN and CapsNet is significantly better than using a $\mathrm{CNN}$ alone when only small datasets are used for training. We have also proven that the in-depth learning model we proposed does not rely on pre-training or data augmentation, which greatly reduces the training time. Faced with a novel coronavirus pneumonia pandemic, high accuracy and low time consumption are obviously very beneficial in the large-scale detection of COVID-19. This also proves that it is possible to use a deep learning model for the large-scale screening and diagnosis of COVID-19 from X-ray images. We sincerely hope that the epidemic will pass as soon as possible and people will return to their previous lives. 


\section{Declarations}

\section{1. [Acknowledgements]}

Thank you very much for the novel coronavirus pneumonia chest X-ray image dataset COVIDx provided by Linda Wang et al. This is really an amazing work, which enables us to continue our research on novel coronavirus pneumonia's related contents.

\section{Funding}

The authors state that this work has not received any funding.

\section{Compliance with Ethical Standards}

\section{Guarantor:}

The scientific guarantor of this publication is Xiaoyu Cui.

\section{Conflict of Interest:}

The authors of this manuscript declare no relationships with any companies, whose products or services may be related to the subject matter of the article.

\section{Competing Interests:}

The authors declare no competing interests.

\section{Statistics and Biometry:}

No complex statistical methods were necessary for this paper.

\section{Informed Consent:}

Written informed consent was obtained from all subjects (patients) in this study.

\section{Ethical Approval:}

Institutional Review Board approval was obtained.

\section{Study subjects or cohorts overlap:}

No

\section{Methodology}

Methodology: 
- retrospective

- diagnostic or prognostic study

- multicenter study

\section{Abbreviations}

\begin{tabular}{ll} 
SARS-CoV-2 & Severe Acute Respiratory Syndrome Coronavirus type 2 \\
\hline COVID-19 & Corona Virus Disease 2019 \\
\hline RT-PCR & Reverse Transcription-Polymerase Chain Reaction \\
\hline CT & Computed Tomography \\
\hline CNN & Convolutional neural network \\
\hline TP & True Positives \\
\hline FP & False Positives \\
\hline TN & True Negatives \\
\hline FN & False Negatives \\
\hline PPV & Positive Predictive Value
\end{tabular}

\section{References}

[1] Timeline of WHO's response to COVID-19. World Health Organization Web site. Available via https://www.who.int/news-room/detail/29-06-2020-covidtimeline. Accessed 30 Aug 2020 [2] WHO Coronavirus Disease (COVID-19) Dashboard. World Health Organization Web site. Available via https://covid19.who.int/. Accessed 30 Aug 2020

[3] Wang W , Xu Y, Gao R , et al (2020) Detection of SARS-CoV-2 in Different Types of Clinical Specimens. JAMA The Journal of the American Medical Association. https://doi.org/10.1001/jama.2020.3786 [4] Corman VM, Landt O, Kaiser M et al (2020) Detection of 2019 novel coronavirus (2019-nCoV) by realtime RT-PCR. EUROSURVEILLANCE. 25(3):2000045

[5] Ai T, Yang Z, Hou H et al (2020) Correlation of Chest CT and RT-PCR Testing for Coronavirus Disease 2019 (COVID-19) in China: A Report of 1014 Cases. Radiology. 296:E32-E40

[6] Kong W (2020) Chest Imaging Appearance of COVID-19 Infection. Radiology Cardiothoracic Imaging. 2:e200028.

[7] Shi $\mathrm{H}$, Han X, Jiang $\mathrm{N}$ et al (2020) Radiological findings from 81 patients with COVID-19 pneumonia in Wuhan, China: a descriptive study. The Lancet Infect Dis. 20:425-434

[8] Xie X, Zhong Z, Zhao W, Zheng C, Wang F, Liu J (2020) Chest CT for Typical 2019-nCoV Pneumonia: Relationship to Negative RT-PCR Testing. Radiology. 296:200343 
[9] Wong HYF, Lam HYS, Fong AH et al (2020) Frequency and Distribution of Chest Radiographic Findings in Patients Positive for COVID-19. Radiology. 296:E72-E78

[10] Greenspan H, Van Ginneken B, Summers RM (2016) Guest editorial deep

learning in medical imaging: Overview and future promise of an exciting new technique. IEEE Trans Med Imaging. 35:1153-1159

[11] Wang L, Wong A (2020) COVID-Net: A Tailored Deep Convolutional Neural Network Design for Detection of COVID-19 Cases from Chest X-Ray Images. arXiv preprint arXiv:2003.09871

[12] A. Narin, C. Kaya, and Z. Pamuk (2020) Automatic Detection of Coronavirus Disease (COVID-19)

Using X-ray Images and Deep Convolutional Neural Networks. arXiv preprint arXiv:2003.10849

[13] Apostolopoulos ID, Bessiana T (2020) Covid-19: automatic detection from X-ray images utilizing transfer learning with convolutional neural networks. Phys Eng Sci Med. 43:635-640

[14] E. El-Din Hemdan, M. A. Shouman, and M. Esmail Karar (2020) COVIDX-net: A framework of deep learning classifiers to diagnose COVID-19 in X-ray images. arXiv preprint arXiv:2003.11055

[15] I. D. Apostolopoulos, S. Aznaouridis, and M. Tzani (2020) Extracting possibly representative COVID19 biomarkers from $X$-ray images with deep learning approach and image data related to pulmonary diseases. arXiv preprint arXiv:2004.00338

[16] Farooq M, Hafeez A (2020) COVID-ResNet: A Deep Learning Framework for Screening of COVID19 from Radiographs. arXiv preprint arXiv:2003.14395

[17] Sabour S, Frosst N, Hinton GE (2017) Dynamic Routing Between Capsules. In Advances in Neural Information Processing Systems. pages 3856-3866

[18] Afshar P, Heidarian S, Naderkhani F, Oikonomou A, Plataniotis K, Mohammadi A (2020) COVID-CAPS: A Capsule Network-based Framework for Identification of COVID-19 cases from X-ray Images. arXiv preprint arXiv:2004.02696

[19] G. Huang, Z. Liu, L. Van Der Maaten and K. Q. Weinberger (2017) Densely Connected Convolutional Networks. IEEE Conference on Computer Vision and Pattern Recognition (CVPR). pp. 2261-2269 [20] He K, Zhang X, Ren S, Sun J (eds) (2016) Deep residual learning for image recognition. Proceedings of the IEEE conference on computer vision and pattern recognition [21] A. Krizhevsky, I. Sutskever, G. E. Hinton (2012) ImageNet Classification with Deep Convolutional Neural Networks. Neural Information Processing Systems (NIPS) [22] M. Chowdhury, T. Rahman, A. Khandakar, et al (2020) Can Al help in screening viral and COVID-19 pneumonia? arXiv preprint arXiv:2003.13145

[23] Minaee S, Kafieh R, Sonka M, Yazdani S, Soufi GJ (2020) Deep-COVID: Predicting COVID-19 From Chest X-Ray Images Using Deep Transfer Learning. Medical Image Analysis.

https://doi.org/10.1016/j.media.2020.101794

\section{Tables}

Table 1 Comparison of CNNs and CNNs Combined with CapsNet 


\begin{tabular}{|lllll|}
\hline Architecture & Accuracy & Precision & Sensitivity & Specificity \\
\hline ResNet50 & $78.28 \%$ & $91.11 \%$ & $59.42 \%$ & $94.89 \%$ \\
\hline DenseNet121 & $87.78 \%$ & $93.71 \%$ & $79.23 \%$ & $95.32 \%$ \\
\hline ResCapsNet & $96.38 \%$ & $94.84 \%$ & $97.58 \%$ & $95.32 \%$ \\
\hline DenseCapsNet & $99.32 \%^{*}$ & $100 \%^{*}$ & $98.55 \%^{*}$ & $100 \%{ }^{*}$ \\
\hline
\end{tabular}

Table 2 Whether DenseCapsNet depends on the experimental results generated by data augmentation and pre-training is tested, and the experimental results of COVID-CAPS are compared.

\begin{tabular}{|llllc|}
\hline Architecture & Accuracy & Precision & Sensitivity & Specificity \\
\hline DenseCapsNet (No Data Augmentation) & $99.10 \%$ & $98.10 \%$ & $100 \%^{*}$ & $98.30 \%$ \\
\hline DenseCapsNet (No Pre-training) & $98.64 \%$ & $99.02 \%$ & $98.07 \%$ & $99.15 \%^{*}$ \\
\hline $\begin{array}{l}\text { DenseCapsNet (No Pre-training or Data } \\
\text { Augmentation) }\end{array}$ & $99.32 \%^{*}$ & $99.04 \%^{*}$ & $99.52 \%$ & $99.15 \%^{*}$ \\
\hline COVID-CAPS & $95.70 \%$ & & $90.00 \%$ & $95.80 \%$ \\
\hline
\end{tabular}

Table 3 Results of comparison between DenseCapsNet and COVID-Net in the ability to detect three classifications

\begin{tabular}{|lllll|}
\hline \multirow{3}{*}{ Sensitivity } & Architecture & Normal & Non-COVID & COVID-19 \\
\cline { 2 - 5 } & COVID-Net & $95.00 \%$ & $94.00 \%$ & $91.00 \%$ \\
\cline { 2 - 5 } & DenseCapsNet & $96.17 \%{ }^{*}$ & $95.32 \%^{*}$ & $99.52 \%{ }^{*}$ \\
\hline \multirow{2}{*}{ PPV } & COVID-Net & $90.50 \%$ & $91.30 \%$ & $98.90 \%{ }^{*}$ \\
\cline { 2 - 5 } & DenseCapsNet & $97.00 \%{ }^{*}$ & $96.55 \%{ }^{*}$ & $97.17 \%$ \\
\hline
\end{tabular}

Note: PPV Positive Predictive Value

\section{Figures}


Normal

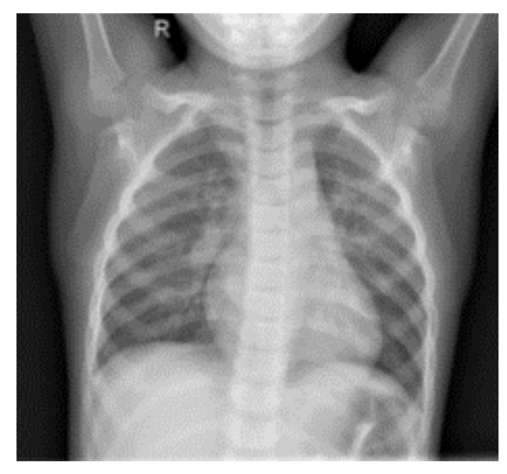

Bacterial Pneumonia

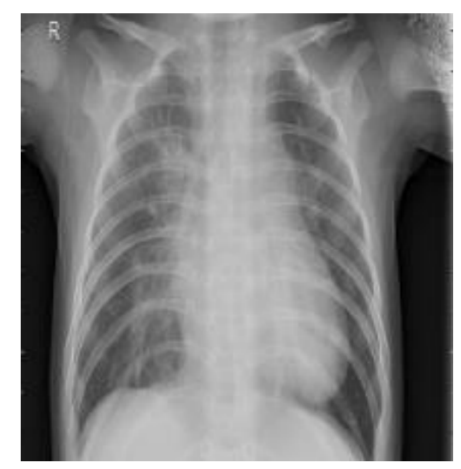

Viral Pneumonia

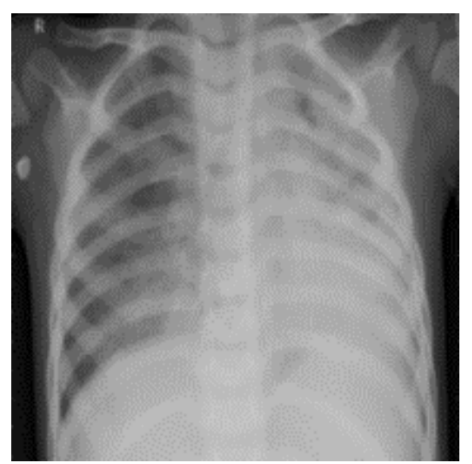

COVID-19

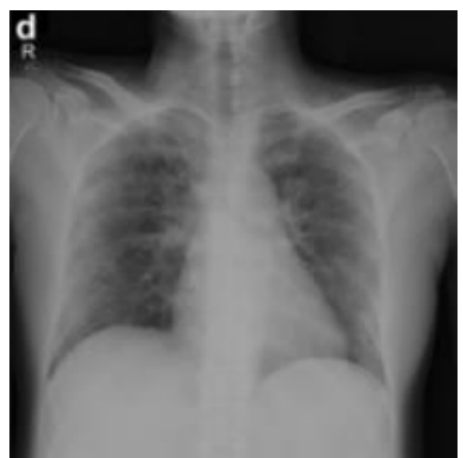

\section{Figure 1}

X-ray examples of normal subjects and bacterial pneumonia, viral pneumonia and COVID-19 patients

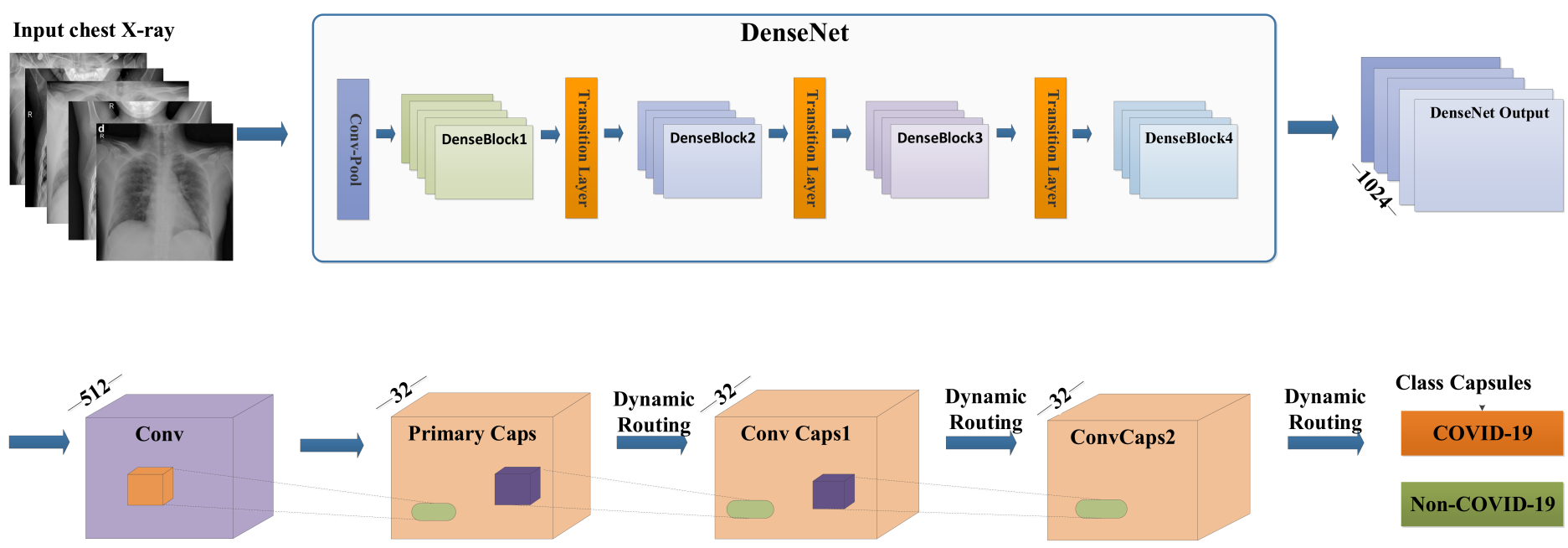

Figure 2

Framework structure of DenseCapsNet; the whole structure is divided into two parts. The front is the feature extraction part, which is DenseNet121. The back part uses the capsule network to find the spatial relationships of the features and finally obtains accurate predictions of COVID-19. 

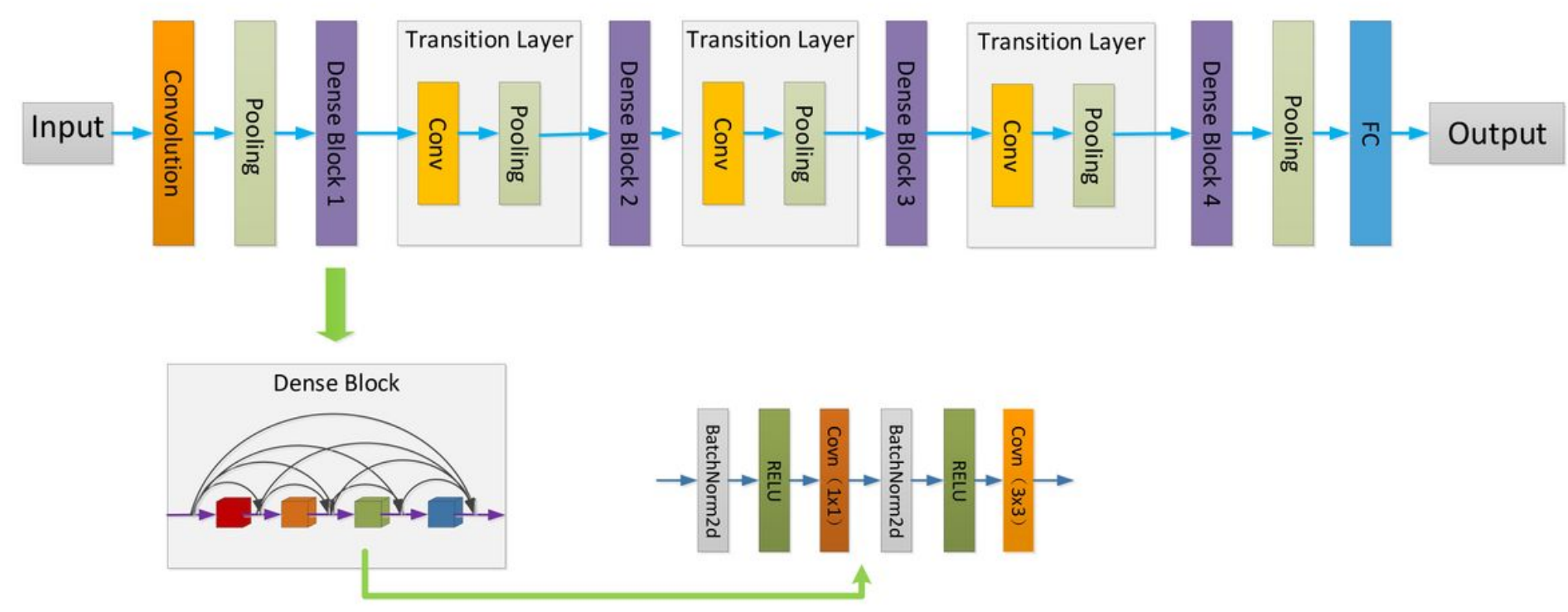

Figure 3

DenseNet structure diagram. Dense Block and Transition Layer are the main structures of DenseNet. Dense Block can make the network structure better for feature transfer and feature reuse. Transition Layer can reduce the size of the feature graph and promote the feature transfer between adjacent Dense Blocks. Finally, the information transmission of the network will be more intensive. 


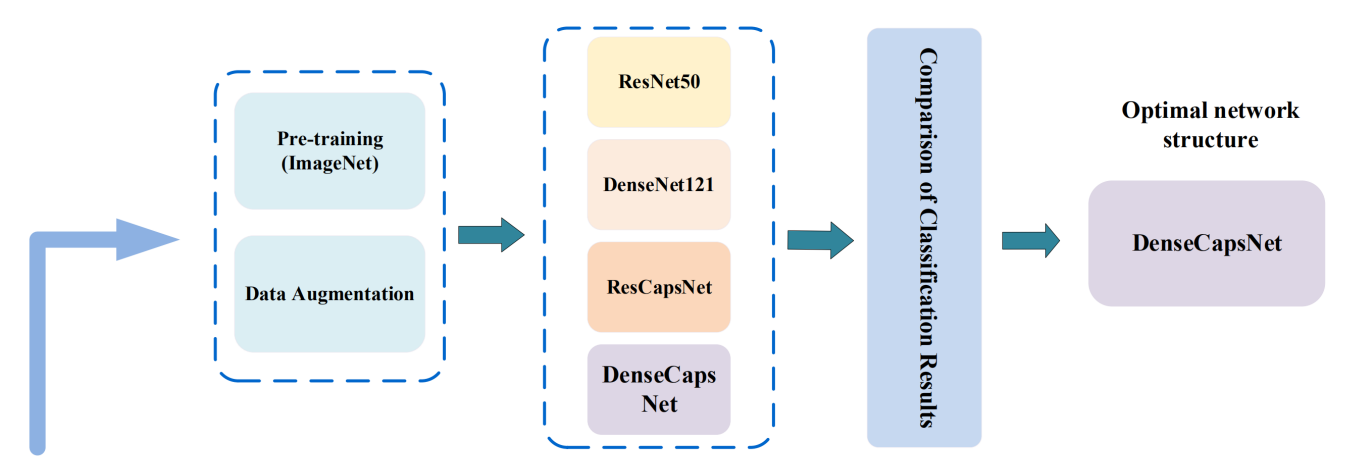

Experiment I
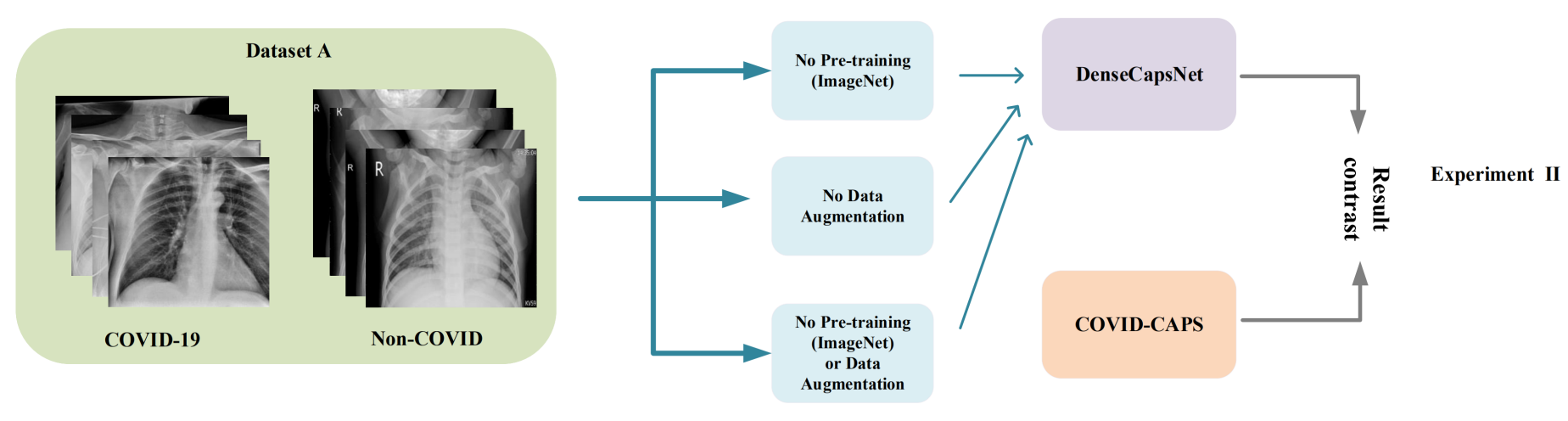

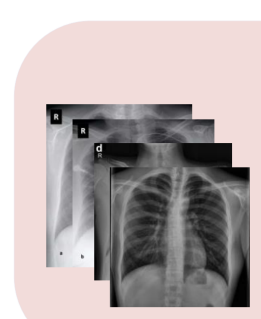

COVID-19

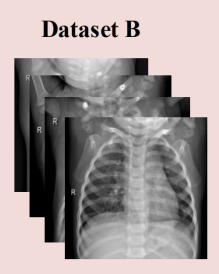

Normal

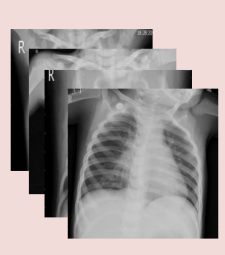

Pneumonia

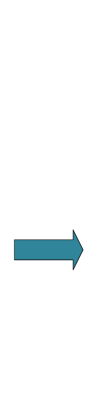

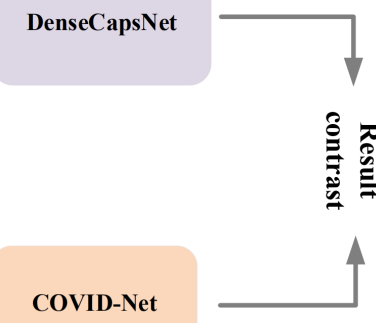

\section{Figure 4}

We tested DenseCapsNet's performance in detecting COVID-19 through three sets of experiments. Experiment I determined that the CNN combined with the capsule network can have a better ability to detect COVID-19 by comparing the performance differences between the CNN, ResCapsNet and DenseCapsNet, and we determined that DenseCapsNet was the best choice. In Experiment II, the effects of COVID-CAPS on DenseCapsNet without data augmentation or pre-training were evaluated, and the final results were compared with those of COVID-CAPS. In Experiment III, DenseCapsNet was trained with three classification datasets for comparison with COVID-Net. 

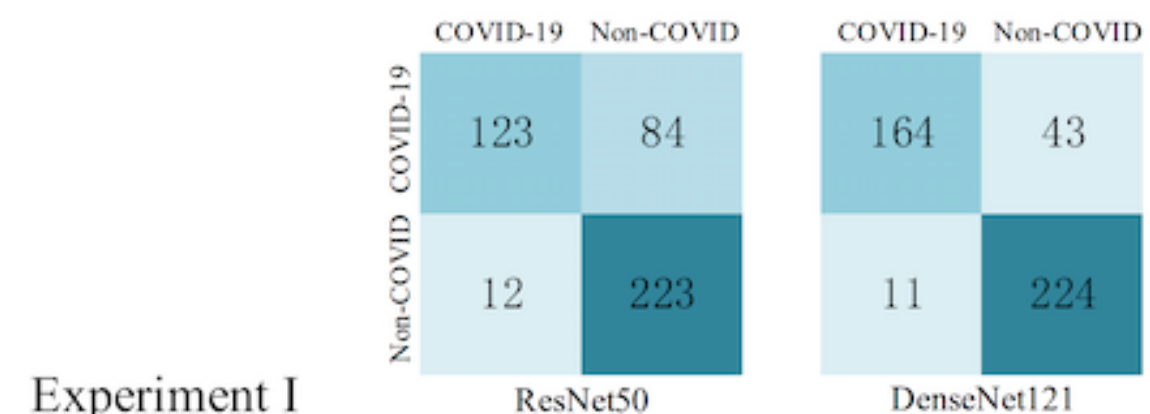

DenseNet121

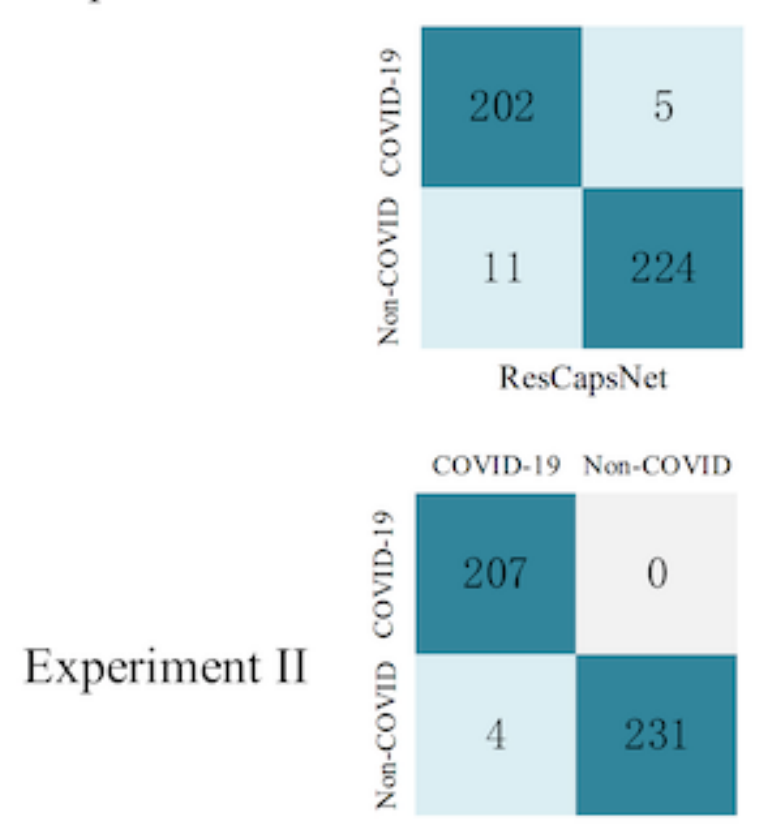

A

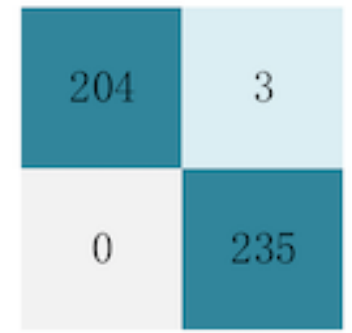

DenseCapsNet
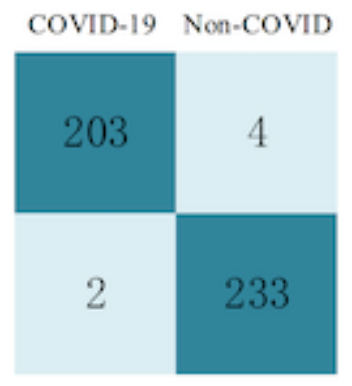

$\mathrm{B}$

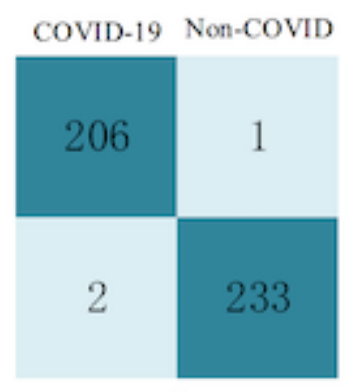

C

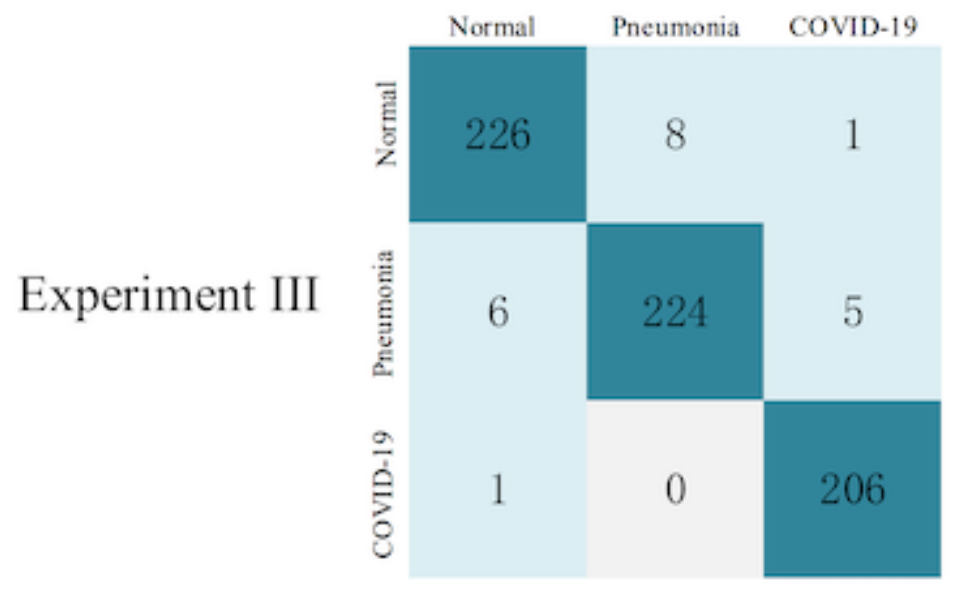

\section{Figure 5}

Experiment I: We use ResNet, DenseNet121, ResCapsNet and DenseCapsNet depth learning frameworks to detect COVID-19 chest X-ray images and obtain confusion matrix results. Experiment II: The influence of data augmentation and pre-training on DenseCapNet is discussed, A does not use data augmentation; $B$ does not use pre-training; $C$ uses neither data augmentation nor pre-training. Experiment III: Confusion 
matrix of chest X-rays for COVID-19, non-COVID-19 pneumonia and normal images detected by DenseCapsNet 